\title{
Hot and sour: the challenges of reconstructing ocean acidification associated with hyperthermals using boron isotopes \\ GAVIN L FOSTER
}

University of Southampton

Presenting Author: gavin.foster@noc.soton.ac.uk

There are few, if any, direct analogues for anthropogenic climate change in the geological record. This is because it is occurring at a pace that is rarely seen naturally, short of those rare times when the Earth was hit by an asteroid (e.g. 66 million years ago). There are events that occur naturally that are however relatively similar - these are known as the "hyperthermals". Hyperthermals are geological rapid, relatively short events $(<1$ million years) characterised by rapid warming and caused by the injection of carbon to the climate system, typically a doubling or more of atmospheric $\mathrm{CO} 2$ levels. It has also recently become apparent that not only do many hyperthermals share similar characteristics (rapid warming, ocean acidification, ocean anoxia, etc), the majority (all?) of the major extinction events in geological history are associated with them.

Despite this clear importance of hyperthermals for understanding the climatic, environmental and biological evolution of the Earth, they represent a significant challenge as the rate and true magnitude of change are difficult to reconstruct using the normal geological tools at our disposal. In this contribution I will discuss these issues and, by focusing on the Palaeocene-Eocene Thermal Maximum, present some solutions exploiting the latest developments in boron isotope analytical methodologies. 